\title{
OPTIMAL ORDER YIELDING DISCREPANCY PRINCIPLE FOR SIMPLIFIED REGULARIZATION IN HILBERT SCALES: FINITE-DIMENSIONAL REALIZATIONS
}

\author{
SANTHOSH GEORGE and M. THAMBAN NAIR
}

Received 13 June 2003

\begin{abstract}
Simplified regularization using finite-dimensional approximations in the setting of Hilbert scales has been considered for obtaining stable approximate solutions to ill-posed operator equations. The derived error estimates using an a priori and a posteriori choice of parameters in relation to the noise level are shown to be of optimal order with respect to certain natural assumptions on the ill posedness of the equation. The results are shown to be applicable to a wide class of spline approximations in the setting of Sobolev scales.
\end{abstract}

2000 Mathematics Subject Classification: 65R10, 65J10, 46E35, 47A50.

1. Introduction. Many of the inverse problems that occur in science and engineering are ill posed, in the sense that a unique solution that depends continuously on the data does not exist. A typical example of an ill-posed equation that often occurs in practical problems, such as in geological prospecting, computer tomography, steel industry, and so forth, is the Fredholm integral equation of the first kind (cf. [2, 6, 8]). Many such problems can be put in the form of an operator equation $A x=y$, where $A: X \rightarrow Y$ is a bounded linear operator between Hilbert spaces $X$ and $Y$ with its range $R(A)$ not closed in $Y$.

Regularization methods are to be employed for obtaining a stable approximate solution for an ill-posed problem. Tikhonov regularization is a simple and widely used procedure to obtain stable approximate solutions to an ill-posed operator equation (2.1). In order to improve the error estimates available in Tikhonov regularization, Natterer [17] carried out error analysis in the framework of Hilbert scales. Subsequently, many authors extended, modified, and generalized Natterer's work to obtain error bounds under various contexts (cf. Neubauer [18], Hegland [7], Schröter and Tautenhahn [20], Mair [10], Nair et al. [16], and Nair [13, 15]). Finite-dimensional realizations of the Hilbert scales approach has been considered by Engl and Neubauer [3].

If $Y=X$ and $A$ itself is a positive selfadjoint operator, then the simplified regularization introduced by Lavrentiev is better suited than Tikhonov regularization in terms of speed of convergence and condition numbers of the resulting equations in the case of finite-dimensional approximations (cf. Schock [19]).

In [4], the authors introduced the Hilbert scales variant of the simplified regularization and obtained error estimates under a priori and a posteriori parameter choice strategies which are optimal in the sense of the "best possible worst error" with respect to certain source set. Recently (cf. [5]), the authors considered a new discrepancy 
principle yielding optimal rates which does not involve certain restrictive assumptions as in [4]. The purpose of this paper is to obtain a finite-dimensional realization of the results in [5].

2. Preliminaries. Let $H$ be a Hilbert space and $A: H \rightarrow H$ a positive, bounded selfadjoint operator on $H$. The inner product and the corresponding norm are denoted by $\langle\cdot, \cdot\rangle$ and $\|\cdot\|$, respectively. Recall that $A$ is said to be a positive operator if $\langle A x, x\rangle \geq 0$ for every $x \in H$. For $y \in R(A)$, the range of $A$, consider the operator equation

$$
A x=y .
$$

Let $\hat{x}$ be the minimal norm solution of (2.1). It is well known that if $R(A)$ is not closed in $H$, then the problem of solving (2.1) for $\hat{x}$ is ill posed in the sense that small perturbations in the data $y$ can cause large deviations in the solution. A prototype of an ill-posed equation (2.1) is an integral equation of the first kind,

$$
\int_{0}^{1} k(\xi, t) x(t) d t=y(\xi), \quad 0 \leq \xi \leq 1,
$$

where $k(\cdot, \cdot)$ is a nondegenerate kernel which is square integrable, that is,

$$
\int_{0}^{1} \int_{0}^{1}|k(\xi, t)|^{2} d t d \xi<\infty,
$$

satisfying $k(\xi, t)=k(t, \xi)$ for all $\xi, t$ in $[0,1]$, and such that the eigenvalues of the corresponding integral operator $A: L^{2}[0,1] \rightarrow L^{2}[0,1]$,

$$
(A x)(\xi)=\int_{0}^{1} k(\xi, t) x(t) d t, \quad 0 \leq \xi \leq 1,
$$

are all nonnegative (cf. [14]). For example, one of the important ill-posed problems which arise in applications is the backward heat equation problem: the problem is to determine the initial temperature $\varphi_{0}:=u(\cdot, 0)$ from the measurements of the final temperature $\varphi_{T}:=u(\cdot, T)$, where $u(\xi, t)$ satisfies

$$
\begin{gathered}
u_{t}-u_{\xi \xi}=0, \quad(\xi, t) \in(0,1) \times(0, T), \\
u(0, t)=u(1, t)=0, \quad t \in[0, T] .
\end{gathered}
$$

We recall from elementary theory of partial differential equations that the solution $u(\xi, t)$ of the above heat equation is given by (cf. Weinberger [23])

$$
u(\xi, t)=\sum_{n=1}^{\infty} \hat{\varphi}_{0}(n) e^{-n^{2} \pi^{2} t} \sin (n \pi \xi),
$$

where $\hat{\varphi}_{0}(n)$ for $n \in \mathbb{N}$ are the Fourier coefficients of the initial temperature $\varphi_{0}(\xi):=$ $u(\xi, 0)$. Hence,

$$
u(\xi, T)=\sum_{n=1}^{\infty} \hat{\varphi}_{0}(n) e^{-n^{2} \pi^{2} T} \sin (n \pi \xi) .
$$


The above equation can be written as

$$
\varphi_{T}(s)=\sum_{n=1}^{\infty} e^{-n^{2} \pi^{2} T}\left\langle\varphi_{0}, u_{n}\right\rangle u_{n}(\xi) \quad \text { with } u_{n}(\xi)=\sqrt{2} \sin (n \pi \xi) .
$$

Thus the problem is to solve the operator equation

$$
A \varphi_{0}=\varphi_{T}
$$

where $A: L^{2}[0,1] \rightarrow L^{2}[0,1]$ is the operator defined by

$$
(A \varphi)(\xi)=\sum_{n=1}^{\infty} e^{-n^{2} \pi^{2} T}\left\langle\varphi, u_{n}\right\rangle u_{n}(\xi)=\int_{0}^{1} k(\xi, t) \varphi(t) d t, \quad 0 \leq \xi \leq 1,
$$

where

$$
k(\xi, t):=\sum_{n=1}^{\infty} e^{-n^{2} \pi^{2} T} u_{n}(\xi) u_{n}(t) .
$$

Note that the above integral operator is compact, positive, and selfadjoint with positive eigenvalues $e^{-n^{2} \pi^{2} T}$ and corresponding eigenvectors $u_{n}(\cdot)$ for $n \in \mathbb{N}$.

For considering the regularization of (2.1) in the setting of Hilbert scales, we consider a Hilbert scale $\left\{H_{t}\right\}_{t \in \mathbb{R}}$ generated by a strictly positive operator $L: D(L) \rightarrow H$ with its domain $D(L)$ dense in $H$ satisfying

$$
\|L x\| \geq\|x\|, \quad x \in D(L) .
$$

By the operator $L$ being strictly positive, we mean that $\langle L x, x\rangle>0$ for all nonzero $x \in H$. Recall (cf. [9]) that the space $H_{t}$ is the completion of $D:=\bigcap_{k=0}^{\infty} D\left(L^{k}\right)$ with respect to the norm $\|x\|_{t}$, induced by the inner product

$$
\langle u, v\rangle_{t}=\left\langle L^{t} u, L^{t} v\right\rangle, \quad u, v \in D .
$$

Moreover, if $\beta \leq \gamma$, then the embedding $H_{\gamma} \hookrightarrow H_{\beta}$ is continuous, and therefore the norm $\|\cdot\|_{\beta}$ is also defined in $H_{\gamma}$ and there is a constant $c_{\beta, \gamma}$ such that

$$
\|x\|_{\beta} \leq c_{\beta, \gamma}\|x\|_{\gamma} \quad \forall x \in H_{\beta} .
$$

An important inequality that we require in the analysis is the interpolation inequality

$$
\|x\|_{\lambda} \leq\|x\|_{r}^{\theta}\|x\|_{t}^{1-\theta}, \quad x \in H_{t},
$$

where

$$
r \leq \lambda \leq t, \quad \theta=\frac{t-\lambda}{t-r}
$$

and the moment inequality

$$
\left\|B^{u} x\right\| \leq\left\|B^{v} x\right\|^{u / v}\|x\|^{1-u / v}, \quad 0 \leq u \leq v,
$$

where $B$ is a positive selfadjoint operator (cf. [2]). 
We assume that the ill-posed nature of the operator $A$ is related to the Hilbert scale $\left\{H_{t}\right\}_{t \in \mathfrak{R}}$ according to the relation

$$
c_{1}\|x\|_{-a} \leq\|A x\| \leq c_{2}\|x\|_{-a}, \quad x \in H,
$$

for some positive reals $a, c_{1}$, and $c_{2}$.

For the example of the integral operator considered in (2.4), one may take $L$ to be defined by

$$
L x:=\sum_{j=1}^{\infty} j^{2}\left\langle x, u_{j}\right\rangle u_{j}
$$

where $u_{j}(t):=\sqrt{2} \sin (j \pi t), j \in \mathbb{N}$ with domain of $L$ as

$$
D(L):=\left\{x \in L^{2}[0,1]: \sum_{j=1}^{\infty} j^{4}\left|\left\langle x, u_{j}\right\rangle\right|^{2}<\infty\right\} .
$$

In this case, it can be seen that

$$
H_{t}=\left\{x \in L^{2}[0,1]: \sum_{j=1}^{\infty} j^{4 t}\left|\left\langle x, u_{j}\right\rangle\right|^{2}<\infty\right\}
$$

and the constants $a, c_{1}$, and $c_{2}$ in (2.18) are given by $a=1, c_{1}=c_{2}=1 / \pi^{2}$ (see Schröter and Tautenhahn [20, Section 4]).

The regularized approximation of $\hat{x}$, considered in [4] is the solution of the wellposed equation

$$
\left(A+\alpha L^{s}\right) x_{\alpha}=y, \quad \alpha>0
$$

where $s$ is a fixed nonnegative real number. Note that if $D(L)=X$ and $L=I$, then the above procedure is the simplified or Lavrentiev regularization.

Suppose the data $y$ is known only approximately, say $\tilde{y}$ in place of $y$ with $\|y-\tilde{y}\| \leq \delta$ for a known error level $\delta>0$. Then, in place of (2.22), we have

$$
\left(A+\alpha L^{s}\right) \tilde{x}_{\alpha}=\tilde{y} .
$$

It can be seen that the solution $\tilde{x}_{\alpha}$ of the above equation is the unique minimizer of the function

$$
x \longmapsto\langle A x, x\rangle-2\langle\tilde{y}, x\rangle+\alpha\left\langle L^{s} x, x\right\rangle, \quad x \in D(L)
$$

One of the crucial results for proving the results in $[4,5]$ as well as the results in this paper is the following proposition, where the functions $f$ and $g$ are defined by

$$
f(t)=\min \left\{c_{1}^{t}, c_{2}^{t}\right\}, \quad g(t)=\max \left\{c_{1}^{t}, c_{2}^{t}\right\}, \quad t \in \mathbb{R},|t| \leq 1,
$$

respectively, with $c_{1}, c_{2}$ as in (2.18). 
Proposition 2.1 (cf. [4, Proposition 3.1]). For $s>0$ and $|v| \leq 1$,

$$
f\left(\frac{v}{2}\right)\|x\|_{-v(s+a) / 2} \leq\left\|A_{s}^{v / 2} x\right\| \leq g\left(\frac{v}{2}\right)\|x\|_{-v(s+a) / 2}, \quad x \in H,
$$

where $A_{s}=L^{-s / 2} A L^{-s / 2}$.

Using the above proposition, the following result has been proved by George and Nair [4].

TheOrem 2.2 (cf. [4, Theorem 3.2]). Suppose $\hat{x} \in H_{t}, 0<t \leq s+a$, and $\alpha>0$, and $\tilde{x}_{\alpha}$ is as in (2.23). Then

$$
\left\|\hat{x}-\tilde{x}_{\alpha}\right\| \leq \phi(s, t) \alpha^{t /(s+a)}\|\hat{x}\|_{t}+\psi(s) \alpha^{-a /(s+a)} \delta
$$

where

$$
\phi(s, t)=\frac{g((s-2 t) /(2 s+2 a))}{f(s /(2 s+2 a))}, \quad \psi(s)=\frac{g(-s /(2 s+2 a))}{f(s /(2 s+2 a))} .
$$

In particular, if $\alpha=c_{0} \delta^{(s+a) /(t+a)}$ for some constant $c_{0}>0$, then

$$
\left\|\hat{x}-\tilde{x}_{\alpha}\right\| \leq \eta(s, t) \delta^{t /(t+a)},
$$

where

$$
\eta(s, t)=\max \left\{\phi(s, t)\|x\|_{t} c_{0}^{t /(t+a)}, \psi(s) c_{0}^{-a /(s+a)}\right\}
$$

For proposing a finite-dimensional realization, we consider a family $\left\{S_{h}: h>0\right\}$ of finite-dimensional subspaces of $H_{k}$ for some $k \geq s$, and consider the minimizer $\tilde{x}_{\alpha, h}$ of the map defined in (2.24) when $x$ varies over $S_{h}$. Equivalently, $\tilde{x}_{\alpha, h}$ is the unique element in $S_{h}$ satisfying the equation

$$
\left\langle\left(A+\alpha L^{s}\right) \tilde{x}_{\alpha, h}, \varphi\right\rangle=\langle\tilde{y}, \varphi\rangle \quad \forall \varphi \in S_{h}
$$

As in Engl and Neubauer [3], we assume the following approximation properties for $S_{h}$.

There exists a constant $\kappa>0$ such that for every $u \in H_{r}$ with $r>k \geq s$,

$$
\inf \left\{\|u-\varphi\|_{k}: \varphi \in S_{h}\right\} \leq \kappa h^{r-k}\|u\|_{r}, \quad h>0 .
$$

As already exemplified in [3], the above assumption is general enough to include a wide variety of approximations spaces, such as spline spaces and finite element spaces.

We will also make use of the following result from Engl and Neubauer [3, Lemma 2.2].

LEMMA 2.3. Under the assumption (2.32), there exists a constant $c>0$ such that for every $u \in H_{s}$ and $h>0$,

$$
\inf _{\varphi \in S_{h}}\left\{h^{-a / 2}\|u-\varphi\|_{-a / 2}+h^{s / 2}\|u-\varphi\|_{s / 2}\right\} \leq c h^{s}\|u\|_{s} .
$$


3. General error estimates. For a fixed $s>0$, let $\tilde{x}_{\alpha}$ and $\tilde{x}_{\alpha, h}$ be as in (2.23) and (2.31), respectively. We will obtain estimate for $\left\|\tilde{x}_{\alpha}-\tilde{x}_{\alpha, h}\right\|$ so that we get an estimate for $\left\|\hat{x}-\tilde{x}_{\alpha, h}\right\|$ using Theorem 2.2 and the relation

$$
\left\|\hat{x}-\tilde{x}_{\alpha, h}\right\| \leq\left\|\hat{x}-\tilde{x}_{\alpha}\right\|+\left\|\tilde{x}_{\alpha}-\tilde{x}_{\alpha, h}\right\| .
$$

In view of the interpolation inequality (2.15), by taking $\rho=-a / 2, \tau=s / 2$, and $\lambda=0$ in (2.15), we get

$$
\|x\| \leq\|x\|_{-a / 2}^{s /(s+a)}\|x\|_{s / 2}^{a /(s+a)}, \quad x \in H_{s / 2} .
$$

Thus, we can deduce an estimate for $\left\|\tilde{x}_{\alpha}-\tilde{x}_{\alpha, h}\right\|$ once we have estimates for $\| \tilde{x}_{\alpha}-$ $\tilde{x}_{\alpha, h} \|_{-a / 2}$ and $\left\|\tilde{x}_{\alpha}-\tilde{x}_{\alpha, h}\right\|_{s / 2}$. For this purpose, we first prove the following.

LEMMA 3.1. Let $\tilde{x}_{\alpha}$ and $\tilde{x}_{\alpha, h}$ be as in (2.23) and (2.31), respectively. Then

$$
\left\|A^{1 / 2}\left(\tilde{x}_{\alpha}-\tilde{x}_{\alpha, h}\right)\right\|^{2}+\alpha\left\|\tilde{x}_{\alpha}-\tilde{x}_{\alpha, h}\right\|_{s / 2}^{2}=\inf _{\varphi \in S_{h}}\left\{\left\|A^{1 / 2}\left(\tilde{x}_{\alpha}-\varphi\right)\right\|^{2}+\alpha\left\|\tilde{x}_{\alpha}-\varphi\right\|_{s / 2}^{2}\right\} .
$$

Proof. It can be seen (cf. [16]) that

$$
\langle u, v\rangle_{*}:=\langle A u, v\rangle+\alpha\left\langle L^{s} u, v\right\rangle, \quad u, v \in D(L),
$$

defines a complete inner product on $D(L)$. Let $\|\cdot\|_{*}$ be the norm induced by $\langle\cdot, \cdot\rangle_{*}$, that is,

$$
\|u\|_{*}=\left(\langle A u, u\rangle+\alpha\left\langle L^{s} u, u\right\rangle\right)^{1 / 2}=\left(\left\|A^{1 / 2} u\right\|^{2}+\alpha\|u\|_{s / 2}^{2}\right)^{1 / 2} .
$$

Let $X$ be the space $D(L)$ with the inner product $\langle\cdot, \cdot\rangle_{*}$ and let $P_{h}$ be the orthogonal projection of $X$ onto the space $S_{h}$. Then from (2.23) and (2.31) we have

$$
\left\langle\left(A+\alpha L^{s}\right)\left(\tilde{x}_{\alpha}-\tilde{x}_{\alpha, h}\right), \varphi\right\rangle=0 \quad \forall \varphi \in S_{h},
$$

that is,

$$
\left\langle\tilde{x}_{\alpha}-\tilde{x}_{\alpha, h}, \varphi\right\rangle_{*}=0 \quad \forall \varphi \in S_{h}
$$

Hence

$$
P_{h}\left(\tilde{x}_{\alpha}-\tilde{x}_{\alpha, h}\right)=0
$$

so that

$$
\left\|\tilde{x}_{\alpha}-\tilde{x}_{\alpha, h}\right\|_{*}=\inf _{\varphi \in S_{h}}\left\|\left(\tilde{x}_{\alpha}-\tilde{x}_{\alpha, h}\right)-\varphi\right\|_{*}=\inf _{\varphi \in S_{h}}\left\|\tilde{x}_{\alpha}-\varphi\right\|_{*} .
$$

Now the result follows using the definition of $\|\cdot\|_{*}$. 
Next we obtain estimate for $\left\|\tilde{x}_{\alpha}-\tilde{x}_{\alpha, h}\right\|$ using the estimates for $\left\|\tilde{x}_{\alpha}-\tilde{x}_{\alpha, h}\right\|_{-a / 2}$ and $\left\|\tilde{x}_{\alpha}-\tilde{x}_{\alpha, h}\right\|_{s / 2}$. We will use the notation

$$
A_{s}:=L^{-s / 2} A L^{-s / 2}
$$

and observe that for $\alpha>0$,

$$
\left(A+\alpha L^{s}\right) x=L^{s / 2}\left(A_{s}+\alpha I\right) L^{s / 2} x \quad \forall x \in H_{s} .
$$

THEOREM 3.2. Suppose $\hat{x} \in H_{t}$ and assumption (2.32) holds, and let $\tilde{x}_{\alpha}$ and $\tilde{x}_{\alpha, h}$ be as in (2.23) and (2.31), respectively. Then

$$
\begin{aligned}
& \left\|\tilde{x}_{\alpha}-\tilde{x}_{\alpha, h}\right\| \\
& \quad \leq f\left(\frac{1}{2}\right)^{-s /(s+a)} \max \{\mathscr{F}(s, a), \mathscr{G}(s, t, a)\} \Phi(s, h, \alpha) \alpha^{-a /(2 s+2 a)}\left(\frac{\delta}{\alpha}+\alpha^{(t-s) /(s+a)}\right) h^{s},
\end{aligned}
$$

where $f$ and $g$ are as in (2.25), and

$$
\begin{gathered}
\mathscr{F}(s, a)=\frac{g(-s /(2 s+2 a))}{f(-s /(2 s+2 a))}, \quad \mathscr{G}(s, t, a)=\frac{g((s-2 t) /(2 s+2 a))}{f(-s /(2 s+2 a))}\|\hat{x}\|_{t}, \\
\Phi(s, h, \alpha)=c \max \left\{g\left(\frac{1}{2}\right) h^{a / 2}, \alpha^{1 / 2} h^{-s / 2}\right\} .
\end{gathered}
$$

Proof. First we prove

$$
\begin{aligned}
& \left\|\tilde{x}_{\alpha}-\tilde{x}_{\alpha, h}\right\|_{-a / 2} \leq \frac{1}{f(1 / 2)} \Phi(s, h, \alpha) h^{s}\left\|\tilde{x}_{\alpha}\right\|_{s}, \\
& \left\|\tilde{x}_{\alpha}-\tilde{x}_{\alpha, h}\right\|_{s / 2} \leq \Phi(s, h, \alpha) \alpha^{-1 / 2} h^{s}\left\|\tilde{x}_{\alpha}\right\|_{s}, \\
& \left\|\tilde{x}_{\alpha}\right\|_{s} \leq \mathscr{F}(s, a) \alpha^{-1} \delta+\mathscr{G}(s, t, a) \alpha^{(t-s) /(s+a)}
\end{aligned}
$$

with $\mathscr{F}(s, a), \mathscr{G}(s, t, a)$, and $\Phi(s, h, \alpha)$ as in the statement of the theorem.

By Lemma 3.1 and Proposition 2.1, it follows that

$$
\begin{aligned}
& f\left(\frac{1}{2}\right)^{2}\left\|\tilde{x}_{\alpha}-\tilde{x}_{\alpha, h}\right\|_{-a / 2}^{2}+\alpha\left\|\tilde{x}_{\alpha}-\tilde{x}_{\alpha, h}\right\|_{s / 2}^{2} \\
& \quad \leq \inf _{\varphi \in S_{h}}\left\{g\left(\frac{1}{2}\right)^{2}\left\|\tilde{x}_{\alpha}-\varphi\right\|_{-a / 2}^{2}+\alpha\left\|\tilde{x}_{\alpha}-\varphi\right\|_{s / 2}^{2}\right\} .
\end{aligned}
$$

Note that

$$
\begin{aligned}
& g\left(\frac{1}{2}\right)^{2}\left\|\tilde{x}_{\alpha}-\varphi\right\|_{-a / 2}^{2}+\alpha\left\|\tilde{x}_{\alpha}-\varphi\right\|_{s / 2}^{2} \\
& \quad \leq\left[g\left(\frac{1}{2}\right)\left\|\tilde{x}_{\alpha}-\varphi\right\|_{-a / 2}+\alpha^{1 / 2}\left\|\tilde{x}_{\alpha}-\varphi\right\|_{s / 2}\right]^{2}
\end{aligned}
$$


But

$$
\begin{aligned}
g\left(\frac{1}{2}\right)\left\|\tilde{x}_{\alpha}-\varphi\right\|_{-a / 2}+\alpha^{1 / 2}\left\|\tilde{x}_{\alpha}-\varphi\right\|_{s / 2} & \\
& \leq \omega_{h, \alpha, s}\left[h^{-a / 2}\left\|\tilde{x}_{\alpha}-\varphi\right\|_{-a / 2}+h^{s / 2}\left\|\tilde{x}_{\alpha}-\varphi\right\|_{s / 2}\right]
\end{aligned}
$$

where

$$
\omega(h, \alpha, s):=\max \left\{g\left(\frac{1}{2}\right) h^{a / 2}, \alpha^{1 / 2} h^{-s / 2}\right\} .
$$

Hence, by Lemma 2.3, we have

$$
f\left(\frac{1}{2}\right)^{2}\left\|\tilde{x}_{\alpha}-\tilde{x}_{\alpha, h}\right\|_{-a / 2}^{2}+\alpha\left\|\tilde{x}_{\alpha}-\tilde{x}_{\alpha, h}\right\|_{s / 2}^{2} \leq\left(\omega(h, \alpha, s) c h^{s}\left\|\tilde{x}_{\alpha}\right\|_{s}\right)^{2} .
$$

In particular,

$$
\begin{array}{r}
f\left(\frac{1}{2}\right)\left\|\tilde{x}_{\alpha}-\tilde{x}_{\alpha, h}\right\|_{-a / 2} \leq \omega(h, \alpha, s) c h^{s}\left\|\tilde{x}_{\alpha}\right\|_{s}, \\
\alpha^{1 / 2}\left\|\tilde{x}_{\alpha}-\tilde{x}_{\alpha, h}\right\|_{s / 2} \leq \omega(h, \alpha, s) c h^{s}\left\|\tilde{x}_{\alpha}\right\|_{s} .
\end{array}
$$

From these, we obtain (3.14) and (3.15).

Now, to prove (3.16), observe from (2.23) and (3.11) that

$$
\tilde{x}_{\alpha}=L^{-s / 2}\left(A_{s}+\alpha I\right)^{-1} L^{-s / 2} \tilde{y} .
$$

By Proposition 2.1, taking $v=-s /(s+a)$, we have

$$
\begin{aligned}
\| L^{s / 2} & \left(A_{s}+\alpha I\right)^{-1} L^{-s / 2}(\tilde{y}-y) \| \\
& \leq \frac{1}{f(-s /(2 s+2 a))}\left\|A_{s}^{-s /(2 s+2 a)}\left(A_{s}+\alpha I\right)^{-1} L^{-s / 2}(\tilde{y}-y)\right\| \\
& \leq \frac{\left\|\left(A_{s}+\alpha I\right)^{-1}\right\|}{f(-s /(2 s+2 a))}\left\|A_{s}^{-s /(2 s+2 a)} L^{-s / 2}(\tilde{y}-y)\right\| \\
& \leq \frac{\alpha^{-1} g(-s /(2 s+2 a))}{f(-s /(2 s+2 a))}\left\|L^{-s / 2}(\tilde{y}-y)\right\|_{s / 2}
\end{aligned}
$$

so that

$$
\left\|L^{s / 2}\left(A_{s}+\alpha I\right)^{-1} L^{-s / 2}(\tilde{y}-y)\right\| \leq \mathscr{F}(s, a) \alpha^{-1} \delta .
$$

Since $L^{-s / 2} y=A_{s} L^{s / 2} \hat{x}$, we have

$$
\begin{aligned}
& \left\|L^{s / 2}\left(A_{s}+\alpha I\right)^{-1} L^{-s / 2} y\right\| \\
& \quad \leq \frac{1}{f(-s /(2 s+2 a))}\left\|A_{s}^{-s /(2 s+2 a)}\left(A_{s}+\alpha I\right)^{-1} A_{s} L^{s / 2} \hat{x}\right\| \\
& \quad \leq \frac{1}{f(-s /(2 s+2 a))}\left\|\left(A_{s}+\alpha I\right)^{-1} A_{s}^{(a+t) /(a+s)}\right\|\left\|A_{s}^{(s-2 t) /(2 a+2 s)} L^{s / 2} \hat{x}\right\|,
\end{aligned}
$$


where

$$
\left\|A_{s}^{(s-2 t) /(2 a+2 s)} L^{s / 2} \hat{x}\right\| \leq g\left(\frac{s-2 t}{2 s+2 a}\right)\|\hat{x}\|_{t} .
$$

Since

$$
\left\|\left(A_{S}+\alpha I\right)^{-1} A_{S}^{\tau}\right\| \leq \alpha^{T-1}, \quad 0<\tau \leq 1,
$$

it follows from the above relations that

$$
\begin{aligned}
\left\|L^{s / 2}\left(A_{s}+\alpha I\right)^{-1} L^{-s / 2} y\right\| & \leq \frac{g((s-2 t) /(2 s+2 a))\|\hat{x}\|_{t}}{f(-s /(2 s+2 a))} \alpha^{(t-s) /(s+a)} \\
& =\mathscr{G}(s, t, a) \alpha^{(t-s) /(s+a)} .
\end{aligned}
$$

Thus, (3.25) and (3.29) give

$$
\begin{aligned}
\left\|\tilde{x}_{\alpha}\right\|_{s} & \leq\left\|L^{s / 2}\left(A_{s}+\alpha I\right)^{-1} L^{-s / 2} \tilde{y}\right\| \\
& \leq\left\|L^{s / 2}\left(A_{s}+\alpha I\right)^{-1} L^{-s / 2}(\tilde{y}-y)\right\|+\left\|L^{s / 2}\left(A_{s}+\alpha I\right)^{-1} L^{-s / 2} y\right\| \\
& \leq \mathscr{F}(s, a) \alpha^{-1} \delta+\mathscr{G}(s, t, a) \alpha^{(t-s) /(s+a)} .
\end{aligned}
$$

Now, the estimates (3.14) and (3.15) together with the interpolation inequality (3.2) give

$$
\begin{aligned}
\left\|\tilde{x}_{\alpha}-\tilde{x}_{\alpha, h}\right\| & \leq\left\|\tilde{x}_{\alpha}-\tilde{x}_{\alpha, h}\right\|_{-a / 2}^{s /(s+a)}\left\|\tilde{x}_{\alpha}-\tilde{x}_{\alpha, h}\right\|_{s / 2}^{a /(s+a)} \\
& \leq f\left(\frac{1}{2}\right)^{-s /(s+a)} \alpha^{-a / 2(s+a)} \Phi(s, h, \alpha) h^{s}\left\|\tilde{x}_{\alpha}\right\|_{s} .
\end{aligned}
$$

From this, the result follows by making use of the estimate (3.16) for $\tilde{x}_{\alpha}$.

4. A priori error estimates. Now we choose the regularization parameter $\alpha$ and discretization parameter $h$ a priori depending on the noise level $\delta$ such that optimal order $O\left(\delta^{t /(t+a)}\right)$ yields whenever $\hat{x} \in H_{t}$.

THEOREM 4.1. Suppose $\hat{x} \in H_{t}$ with $0<t \leq s+a$ and assumption (2.32) holds. Suppose, in addition, that

$$
\alpha=c_{0} \delta^{(s+a) /(t+a)}, \quad h=d_{0} \delta^{1 /(t+a)}
$$

for some constants $c_{0}, d_{0}>0$. Then, using the notations in Theorems 2.2 and 3.2,

$$
\left\|\hat{x}-\tilde{x}_{\alpha, h}\right\| \leq[\eta(s, t)+\xi(s, t)] \delta^{t /(t+a)},
$$


where

$$
\begin{aligned}
\eta(s, t)= & \max \left\{\phi(s, t)\|x\|_{t} c_{0}^{t /(t+a)}, \psi(s) c_{0}^{-a /(s+a)}\right\}, \\
\xi(s, t)= & c\left[f\left(\frac{1}{2}\right)\right]^{-s /(s+a)} d_{0}^{s}\left(c_{0}^{-1}+c_{0}^{(t-s) /(t+a)}\right) \\
& \times \max \{\mathscr{F}(s, a), \mathscr{G}(s, t, a)\} \max \left\{g\left(\frac{1}{2}\right) d_{0}^{a / 2}, c_{0}^{1 / 2} d_{0}^{-s / 2}\right\} .
\end{aligned}
$$

Proof. Using the choice (4.1), it is seen that

$$
\begin{gathered}
\Phi(s, h, \alpha) \alpha^{-a / 2(s+a)}=c c_{0}^{-a / 2(s+a)} \max \left\{g\left(\frac{1}{2}\right) d_{0}^{a / 2}, c_{0}^{1 / 2} d_{0}^{-s / 2}\right\}, \\
\delta \alpha^{-1} h^{s}=c_{0}^{-1} d_{0}^{s} \delta^{t /(t+a)} \\
\alpha^{(t-s) /(s+a)} h^{s}=c_{0}^{(t-s) /(t+a)} d_{0}^{s} \delta^{t /(t+a)} .
\end{gathered}
$$

Therefore, by Theorem 3.2, we have

$$
\left\|\tilde{x}_{\alpha}-\tilde{x}_{\alpha, h}\right\| \leq \xi(s, t) \delta^{t /(t+a)} .
$$

Also, from Theorem 2.2, we have

$$
\left\|\hat{x}-\tilde{x}_{\alpha}\right\| \leq \eta(s, t) \delta^{t /(t+a)} .
$$

Thus the result follows from the inequality

$$
\left\|\hat{x}-\tilde{x}_{\alpha}\right\| \leq\left\|\hat{x}-\tilde{x}_{\alpha}\right\|+\left\|\tilde{x}_{\alpha}-\tilde{x}_{\alpha, h}\right\| .
$$

REMARK 4.2. We observe that the error bound obtained is of the same order as of Theorem 2.2, and this order is optimal with respect to the source set

$$
M_{\rho, t}=\left\{x \in H_{t}:\|x\|_{t} \leq \rho\right\}
$$

in the sense of the best possible worst error (cf. [4]).

5. Discrepancy principle. In this section, we consider a discrepancy principle to choose the regularization parameter $\alpha$ depending on the noise level $\delta$ and the discretization parameter $h$. This is a finite-dimensional variant of the discrepancy principle considered in [5]. 
We assume throughout that $y \neq 0$. Suppose that $\tilde{y} \in H$ is such that

$$
\|y-\tilde{y}\| \leq \delta
$$

for a known error level $\delta>0$ and $P_{h} \tilde{y} \neq 0$, where $P_{h}$ is the orthogonal projection of $H$ onto $S_{h}$. We assume, throughout this section, that

$$
\left\|A\left(P_{h}-I\right)\right\| \leq c_{3} h, \quad h>0
$$

for some $c_{3}>0$, independent of $h$. Let

$$
R_{\alpha}:=\left(A_{s}+\alpha I\right)^{-1}
$$

We will make use of the relation

$$
\left\|R_{\alpha} A_{s}^{\tau}\right\| \leq \alpha^{\tau-1}, \quad \alpha>0,0<\tau \leq 1
$$

which follows from the spectral properties of the selfadjoint operator $A_{s}, s>0$.

Let $s, a$ be fixed positive real numbers. For $\alpha>0$ and $x \in H$, consider the functions

$$
F(\alpha, x)=\frac{\alpha\left\|R_{\alpha}^{3 / 2} A_{s}^{-s /(2 s+2 a)} L^{-s / 2} P_{h} x\right\|^{2}}{\left\|R_{\alpha}^{2} A_{s}^{-s /(2 s+2 a)} L^{-s / 2} P_{h} x\right\|} .
$$

Note that, by assumption (2.18), $\left\|R_{\alpha}^{2} A_{s}^{-s /(2 s+2 a)} L^{-s / 2} P_{h} x\right\|$ is nonzero for every $x \in H$ with $P_{h} x \neq 0$, so that the function $F(\alpha, x)$ is well defined for all such $x$. We observe that the assumption $P_{h} x \neq 0$ is satisfied for $x \neq 0$ and $h$ small enough, if $P_{h} x \rightarrow x$ as $h \rightarrow 0$ for every $x \in H$.

In the following, we assume that $h$ is such that $P_{h} \tilde{y} \neq 0$.

In order to choose the regularization parameter $\alpha$, we consider the discrepancy principle

$$
F(\alpha, \tilde{y})=b \delta+d h
$$

for some $b, d>0$. In the due course, we will make use of the relation

$$
f\left(\frac{-s}{2 s+2 a}\right)\|x\| \leq\left\|A_{s}^{-s /(2 s+2 a)} L^{-s / 2} x\right\| \leq g\left(\frac{-s}{2 s+2 a}\right)\|x\|
$$

which can easily be derived from Proposition 2.1.

First we prove the monotonicity of the function $F(\alpha, x)$ defined in (5.5). 
THEOREM 5.1. Let $x \in H$ be such that the function $\alpha \mapsto F(\alpha, x)$ for $\alpha>0$ in (5.5) is well defined. Then, $F(\cdot, x)$ is increasing and it is continuously differentiable with $F^{\prime}(\alpha, x) \geq 0$ for all $\alpha>0$. In addition,

$$
\lim _{\alpha \rightarrow 0} F(\alpha, x)=0, \quad \lim _{\alpha \rightarrow \infty} F(\alpha, x)=\left\|A_{s}^{-s /(2 s+2 a)} L^{-s / 2} P_{h} x\right\|
$$

Proof. Using the definition (5.5) of $F(\alpha, \cdot)$, we have

$$
\begin{aligned}
\frac{\partial}{\partial \alpha} & F(\alpha, x) \\
= & \frac{(\partial / \partial \alpha)\left(F^{2}(\alpha, x)\right)}{2 F(\alpha, x)} \\
= & \frac{2 \alpha\left\|R_{\alpha}^{2} A_{s}^{-s /(2 s+2 a)} L^{-s / 2} P_{h} x\right\|^{2}\left\|R_{\alpha}^{3 / 2} A_{s}^{-s /(2 s+2 a)} L^{-s / 2} P_{h} x\right\|^{2}}{2 \alpha\left\|R_{\alpha}^{3 / 2} A_{s}^{-s /(2 s+2 a)} L^{-s / 2} P_{h} x\right\|^{2}} \\
& \times \frac{(\partial / \partial \alpha)\left[\alpha\left\|R_{\alpha}^{3 / 2} A_{s}^{-s /(2 s+2 a)} L^{-s / 2} P_{h} x\right\|^{2}\right]}{\left\|R_{\alpha}^{2} A_{s}^{-s /(2 s+2 a)} L^{-s / 2} P_{h} x\right\|^{3}} \\
& -\frac{\alpha^{2}\left\|R_{\alpha}^{3 / 2} A_{s}^{-s /(2 s+2 a)} L^{-s / 2} P_{h} x\right\|^{4}(\partial / \partial \alpha)\left[\left\|R_{\alpha}^{2} A_{s}^{-s /(2 s+2 a)} L^{-s / 2} P_{h} x\right\|^{2}\right]}{2 \alpha\left\|R_{\alpha}^{3 / 2} A_{s}^{-s /(2 s+2 a)} L^{-s / 2} P_{h} x\right\|^{2}\left\|R_{\alpha}^{2} A_{s}^{-s /(2 s+2 a)} L^{-s / 2} P_{h} x\right\|^{3}} \\
= & \frac{\left\|R_{\alpha}^{2} A_{s}^{-s /(2 s+2 a)} L^{-s / 2} P_{h} x\right\|^{2}(\partial / \partial \alpha)\left[\alpha\left\|R_{\alpha}^{3 / 2} A_{s}^{-s /(2 s+2 a)} L^{-s / 2} P_{h} x\right\|^{2}\right]}{\left\|R_{\alpha}^{2} A_{s}^{-s /(2 s+2 a)} L^{-s / 2} P_{h} x\right\|^{3}} \\
& -\frac{\alpha\left\|R_{\alpha}^{3 / 2} A_{s}^{-s /(2 s+2 a)} L^{-s / 2} P_{h} x\right\|^{2}(\partial / \partial \alpha)\left[\left\|R_{\alpha}^{2} A_{s}^{-s /(2 s+2 a)} L_{s}^{-s /(2 s+2 a)} L_{h}^{-s / 2} P_{h} x\right\|^{3}\right]}{2} .
\end{aligned}
$$

Let $\left\{E_{\lambda}: 0 \leq \lambda \leq a\right\}$ be the spectral family of $A_{s}$, where $a \geq\left\|A_{s}\right\|$. Then

$$
\begin{aligned}
\frac{\partial}{\partial \alpha}( & \left.\alpha\left\|R_{\alpha}^{3 / 2} A_{s}^{-s /(2 s+2 a)} L^{-s / 2} P_{h} x\right\|^{2}\right) \\
& =\frac{\partial}{\partial \alpha} \int_{0}^{a} \frac{\alpha}{\lambda^{s /(s+a)}(\lambda+\alpha)^{3}} d\left\langle E_{\lambda} L^{-s / 2} P_{h} x, L^{-s / 2} P_{h} x\right\rangle \\
& =\int_{0}^{a}\left[\frac{1}{\lambda^{s /(s+a)}(\lambda+\alpha)^{3}}-\frac{3 \alpha}{\lambda^{s /(s+a)}(\lambda+\alpha)^{4}}\right] d\left\langle E_{\lambda} L^{-s / 2} P_{h} x, L^{-s / 2} P_{h} x\right\rangle \\
& =\left\|R_{\alpha}^{3 / 2} A_{s}^{-s /(2 s+2 a)} L^{-s / 2} P_{h} x\right\|^{2}-3 \alpha\left\|R_{\alpha}^{2} A_{s}^{-s /(2 s+2 a)} L^{-s / 2} P_{h} x\right\|^{2} .
\end{aligned}
$$

Similarly, we obtain

$$
\frac{\partial}{\partial \alpha}\left(\left\|R_{\alpha}^{2} A_{s}^{-s /(2 s+2 a)} L^{-s / 2} P_{h} x\right\|\right)=-4\left\|R_{\alpha}^{5 / 2} A_{s}^{-s /(2 s+2 a)} L^{-s / 2} P_{h} x\right\|^{2} .
$$


Therefore, from (5.9), by using (5.10) and (5.11), we get

$$
\begin{aligned}
\frac{\partial}{\partial \alpha} F(\alpha, x)= & \frac{\left\|R_{\alpha}^{2} A_{s}^{-s /(2 s+2 a)} L^{-s / 2} P_{h} x\right\|^{2}}{\left\|R_{\alpha}^{2} A_{s}^{-s /(2 s+2 a)} L^{-s / 2} P_{h} x\right\|^{3}} \\
& \times\left[\left\|R_{\alpha}^{3 / 2} A_{s}^{-s /(2 s+2 a)} L^{-s / 2} P_{h} x\right\|^{2}-3 \alpha\left\|R_{\alpha}^{2} A_{s}^{-s /(2 s+2 a)} L^{-s / 2} P_{h} x\right\|^{2}\right] \\
& +\frac{2 \alpha\left\|R_{\alpha}^{3 / 2} A_{s}^{-s /(2 s+2 a)} L^{-s / 2} P_{h} x\right\|^{2}\left\|R_{\alpha}^{5 / 2} A_{s}^{-s /(2 s+2 a)} L^{-s / 2} P_{h} x\right\|^{2}}{\left\|R_{\alpha}^{2} A_{s}^{-s /(2 s+2 a)} L^{-s / 2} P_{h} x\right\|^{3}}
\end{aligned}
$$

The above equation can be rewritten as

$$
\begin{aligned}
\frac{\partial}{\partial \alpha} F(\alpha, x)= & \frac{\left\|R_{\alpha}^{2} A_{s}^{-s /(2 s+2 a)} L^{-s / 2} P_{h} x\right\|^{2}}{\left\|R_{\alpha}^{2} A_{s}^{-s /(2 s+2 a)} L^{-s / 2} P_{h} x\right\|^{3}} \\
& \times\left[\left\|R_{\alpha}^{3 / 2} A_{s}^{-s /(2 s+2 a)} L^{-s / 2} P_{h} x\right\|^{2}-\alpha\left\|R_{\alpha}^{2} A_{s}^{-s /(2 s+2 a)} L^{-s / 2} P_{h} x\right\|^{2}\right] \\
& +\frac{2 \alpha}{\left\|R_{\alpha}^{2} A_{s}^{-s /(2 s+2 a)} L^{-s / 2} P_{h} x\right\|^{3}} \\
& \times\left[\left\|R_{\alpha}^{3 / 2} A_{s}^{-s /(2 s+2 a)} L^{-s / 2} P_{h} x\right\|^{2}\left\|R_{\alpha}^{5 / 2} A_{s}^{-s /(2 s+2 a)} L^{-s / 2} P_{h} x\right\|^{2}\right. \\
& \left.-\left\|R_{\alpha}^{2} A_{s}^{-s /(2 s+2 a)} L^{-s / 2} P_{h} x\right\|^{4}\right] .
\end{aligned}
$$

Since

$$
\begin{aligned}
& \left\|R_{\alpha}^{3 / 2} A_{s}^{-s /(2 s+2 a)} L^{-s / 2} P_{h} x\right\|^{2} \\
& \quad=\left\langle\left(A_{s}+\alpha I\right)^{-3} A_{s}^{-s /(2 s+2 a)} L^{-s / 2} P_{h} x, A_{s}^{-s /(2 s+2 a)} L^{-s / 2} P_{h} x\right\rangle, \\
& \left\|R_{\alpha}^{2} A_{s}^{-s /(2 s+2 a)} L^{-s / 2} P_{h} x\right\|^{2} \\
& \quad=\left\langle\left(A_{s}+\alpha I\right)^{-3} A_{s}^{-s /(2 s+2 a)} L^{-s / 2} P_{h} x,\left(A_{s}+\alpha I\right)^{-1} A_{s}^{-s /(2 s+2 a)} L^{-s / 2} P_{h} x\right\rangle,
\end{aligned}
$$

we see that

$$
\begin{aligned}
\| R_{\alpha}^{3 / 2} & A_{s}^{-s /(2 s+2 a)} L^{-s / 2} P_{h} x \|^{2} \\
= & \alpha\left\|R_{\alpha}^{2} A_{s}^{-s /(2 s+2 a)} L^{-s / 2} P_{h} x\right\|^{2} \\
& +\left\langle\left(A_{s}+\alpha I\right)^{-3} A_{s}^{-s /(2 s+2 a)} L^{-s / 2} P_{h} x, A_{s}\left(A_{s}+\alpha I\right)^{-1} A_{s}^{-s /(2 s+2 a)} L^{-s / 2} P_{h} x\right\rangle \\
= & \alpha\left\|R_{\alpha}^{2} A_{s}^{-s /(2 s+2 a)} L^{-s / 2} P_{h} x\right\|^{2}+\left\|A_{s}^{a /(2 s+2 a)} R_{\alpha}^{2} L^{-s / 2} P_{h} x\right\|^{2} .
\end{aligned}
$$


Also, we have

$$
\begin{aligned}
\left\|R_{\alpha}^{2} A_{s}^{-s /(2 s+2 a)} L^{-s / 2} P_{h} x\right\|^{4} \\
\quad=\left[\left\langle R_{\alpha}^{2} A_{s}^{-s /(2 s+2 a)} L^{-s / 2} P_{h} x, R_{\alpha}^{2} A_{s}^{-s /(2 s+2 a)} L^{-s / 2} P_{h} x\right\rangle\right]^{2} \\
=\left[\left\langle R_{\alpha}^{3 / 2} A_{s}^{-s /(2 s+2 a)} L^{-s / 2} P_{h} x, R_{\alpha}^{5 / 2} A_{s}^{-s /(2 s+2 a)} L^{-s / 2} P_{h} x\right\rangle\right]^{2} \\
\quad \leq\left\|R_{\alpha}^{3 / 2} A_{s}^{-s /(2 s+2 a)} L^{-s / 2} P_{h} x\right\|^{2}\left\|R_{\alpha}^{5 / 2} A_{s}^{-s /(2 s+2 a)} L^{-s / 2} P_{h} x\right\|^{2} .
\end{aligned}
$$

Hence,

$$
\frac{\partial}{\partial \alpha}(F(\alpha, x)) \geq 0
$$

To prove the last part of the theorem, we observe that

$$
\begin{aligned}
& \alpha^{2}\left\|R_{\alpha}^{2} A_{s}^{-s /(2 s+2 a)} L^{-s / 2} P_{h} x\right\|-F(\alpha, x) \\
& \quad=\frac{\alpha^{2}\left\|R_{\alpha}^{2} A_{s}^{-s /(2 s+2 a)} L^{-s / 2} P_{h} x\right\|^{2}-\alpha\left\|R_{\alpha}^{3 / 2} A_{s}^{-s /(2 s+2 a)} L^{-s / 2} P_{h} x\right\|^{2}}{\left\|R_{\alpha}^{2} A_{s}^{-s /(2 s+2 a)} L^{-s / 2} P_{h} x\right\|} .
\end{aligned}
$$

We note that

$$
\begin{aligned}
& \alpha^{2}\left\|R_{\alpha}^{2} A_{s}^{-s /(2 s+2 a)} L^{-s / 2} P_{h} x\right\|^{2}=\alpha\left\langle R_{\alpha}^{3} A_{s}^{-s /(2 s+2 a)} L^{-s / 2} P_{h} x, \alpha R_{\alpha} A_{s}^{-s /(2 s+2 a)} L^{-s / 2} P_{h} x\right\rangle, \\
& \alpha\left\|R_{\alpha}^{3 / 2} A_{s}^{-s /(2 s+2 a)} L^{-s / 2} P_{h} x\right\|^{2}=\alpha\left\langle R_{\alpha}^{3} A_{s}^{-s /(2 s+2 a)} L^{-s / 2} P_{h} x, A_{s}^{-s /(2 s+2 a)} L^{-s / 2} P_{h} x\right\rangle .
\end{aligned}
$$

Since

$$
\alpha R_{\alpha}-I=A_{s} R_{\alpha}=R_{\alpha} A_{s}
$$

it follows that

$$
\begin{aligned}
\alpha^{2} \| & R_{\alpha}^{2} A_{s}^{-s /(2 s+2 a)} L^{-s / 2} P_{h} x \|-F(\alpha, x) \\
& =\frac{-\alpha\left\langle R_{\alpha}^{3} A_{s}^{-s /(2 s+2 a)} L^{-s / 2} P_{h} x, A_{s} R_{\alpha} A_{s}^{-s /(2 s+2 a)} L^{-s / 2} P_{h} x\right\rangle}{\left\|R_{\alpha}^{2} A_{s}^{-s /(2 s+2 a)} L^{-s / 2} P_{h} x\right\|} \\
& =\frac{-\alpha\left\|A_{s}^{a /(2 s+2 a)} R_{\alpha}^{2} L^{-s / 2} P_{h} x\right\|^{2}}{\left\|R_{\alpha}^{2} A_{s}^{-s /(2 s+2 a)} L^{-s / 2} P_{h} x\right\|} \\
& \leq 0
\end{aligned}
$$

so that

$$
F(\alpha, x) \geq \alpha^{2}\left\|R_{\alpha}^{2} A_{s}^{-s /(2 s+2 a)} L^{-s / 2} P_{h} x\right\| \geq \alpha^{2} \frac{\left\|A_{s}^{-s /(2 s+2 a)} L^{-s / 2} P_{h} x\right\|}{\left(\left\|A_{s}\right\|+\alpha\right)^{2}} .
$$


Also, we have

$$
\begin{aligned}
F(\alpha, x) & =\frac{\alpha\left\langle R_{\alpha} A_{s}^{-s /(2 s+2 a)} L^{-s / 2} P_{h} x, R_{\alpha}^{2} A_{s}^{-s /(2 s+2 a)} L^{-s / 2} P_{h} x\right\rangle}{\left\|R_{\alpha}^{2} A_{s}^{-s /(2 s+2 a)} L^{-s / 2} P_{h} x\right\|} \\
& \leq \alpha\left\|R_{\alpha} A_{s}^{-s /(2 s+2 a)} L^{-s / 2} P_{h} x\right\| .
\end{aligned}
$$

Hence

$$
\left(\frac{\alpha}{\left\|A_{s}\right\|+\alpha}\right)^{2}\left\|A_{s}^{-s /(2 s+2 a)} L^{-s / 2} P_{h} x\right\| \leq F(\alpha, x) \leq \alpha\left\|R_{\alpha} A_{s}^{-s /(2 s+2 a)} L^{-s / 2} P_{h} x\right\| .
$$

From this we can conclude that

$$
\lim _{\alpha \rightarrow 0} F(\alpha, x)=0, \quad \lim _{\alpha \rightarrow \infty} F(\alpha, x)=\left\|A_{s}^{-s /(2 s+2 a)} L^{-s / 2} P_{h} x\right\| .
$$

This completes the proof.

For the next theorem, in addition to (5.1), we assume that the inexact data $\tilde{y}$ satisfies the relation

$$
\left\|A_{s}^{-s /(2 s+2 a)} L^{-s / 2} P_{h} \tilde{y}\right\| \geq b \delta+d h .
$$

This assumption is satisfied for small enough $h$ and $\delta$, if, for example,

$$
(b+\tilde{f}(s)) \delta+\left(d+c_{3} \tilde{f}(s)\|\hat{x}\|\right) h \leq \tilde{f}(s)\|y\|,
$$

where $\tilde{f}(s)=f(-s /(2 s+2 a))$, because

$$
\left\|P_{h} \tilde{y}\right\| \geq\|y\|-\left\|\left(I-P_{h}\right) A \hat{x}\right\|-\delta
$$

and by (5.7),

$$
\left\|A_{s}^{-s /(2 s+2 a)} L^{-s / 2} P_{h} \tilde{y}\right\| \geq \tilde{f}(s)\left\|P_{h} \tilde{y}\right\| .
$$

Now the following theorem is a consequence of Theorem 5.1.

THEOREM 5.2. Assume that (5.1) and (5.26) are satisfied. Then there exists a unique $\alpha:=\alpha(\delta, h)$ satisfying

$$
F(\alpha, \tilde{y})=b \delta+d h
$$

In order to obtain an estimate for the error $\left\|\hat{x}-\tilde{x}_{\alpha, h}\right\|$ with the parameter choice strategy (5.30), we will make use of (3.31). The next lemma gives an error estimate for $\left\|\tilde{x}_{\alpha}\right\|_{s}$ in terms of $\alpha=\alpha(\delta, h), \delta$, and $h$.

LEMMA 5.3. Let $\alpha:=\alpha(\delta, h)$ be the unique solution of (5.30). Then for any fixed $\tau>0$,

$$
\left\|\tilde{x}_{\alpha}\right\|_{s} \leq c_{4}(\delta+h)^{\tau /(\tau+1)} \alpha^{-1}
$$


where

$$
c_{4} \geq \max \left\{b+\tilde{\mathfrak{g}}(s), c+c_{3}\|\hat{x}\| \tilde{\mathfrak{g}}(s)\right\} \tilde{\mathfrak{g}}(s)\|\tilde{y}\|
$$

with $\tilde{\mathfrak{g}}(s):=g(-s /(2 s+2 a))$.

Proof. By (3.30), we have

$$
\left\|\tilde{x}_{\alpha}\right\|_{s} \leq\left\|L^{s / 2} R_{\alpha} L^{-s / 2} \tilde{y}\right\| \leq \tilde{f}^{-1}(s) \alpha^{-1}\left\|\alpha R_{\alpha} A_{s}^{-s /(2 s+2 a)} L^{-s / 2} \tilde{y}\right\| .
$$

To obtain an estimate for $\left\|\alpha R_{\alpha} A_{s}^{-s /(2 s+2 a)} L^{-s / 2} \tilde{y}\right\|$, we will make use of the moment inequality (2.17). Precisely, we use (2.17) with

$$
u=\tau, \quad v=1+\tau, \quad B=\alpha R_{\alpha}, \quad x=\alpha^{1-\tau} R_{\alpha}^{1-\tau} A_{s}^{-s /(2 s+2 a)} L^{-s / 2} \tilde{y} .
$$

Then, since

$$
\|x\| \leq\left\|A_{s}^{-s /(2 s+2 a)} L^{-s / 2} \tilde{y}\right\| \leq g\left(\frac{-s}{2 s+2 a}\right)\|\tilde{y}\|,
$$

we have

$$
\begin{aligned}
\left\|\alpha R_{\alpha} A_{s}^{-s /(2 s+2 a)} L^{-s / 2} \tilde{y}\right\| \\
\quad=\left\|B^{T} x\right\| \leq\left\|B^{\tau+1} x\right\|^{\tau /(\tau+1)}\|x\|^{1 /(\tau+1)} \\
\quad=\left\|\alpha^{2} R_{\alpha}^{2} A_{s}^{-s /(2 s+2 a)} L^{-s / 2} \tilde{y}\right\|^{\tau /(\tau+1)}\left[g\left(\frac{-s}{2 s+2 a}\right)\|\tilde{y}\|\right]^{1 /(\tau+1)} .
\end{aligned}
$$

Further, by (5.21),

$$
\begin{aligned}
\| \alpha^{2} & R_{\alpha}^{2} A_{s}^{-s /(2 s+2 a)} L^{-s / 2} \tilde{y} \| \\
& \leq\left\|\alpha^{2} R_{\alpha}^{2} A_{s}^{-s /(2 s+2 a)} L^{-s / 2}\left(I-P_{h}\right) \tilde{y}\right\|+\left\|\alpha^{2} R_{\alpha}^{2} A_{s}^{-s /(2 s+2 a)} L^{-s / 2} P_{h} \tilde{y}\right\| \\
& \leq \tilde{g}(s)\left\|\left(I-P_{h}\right) \tilde{y}\right\|+F(\alpha, \tilde{y}) \\
& \leq \tilde{g}(s)\left[\left\|\left(I-P_{h}\right)(\tilde{y}-y)\right\|+\left\|\left(I-P_{h}\right) A \hat{x}\right\|\right]+F(\alpha, \tilde{y}) \\
& \leq \tilde{g}(s)\left[\delta+c_{3}\|\hat{x}\| h\right]+F(\alpha, \tilde{y}) .
\end{aligned}
$$

Therefore, if $\alpha:=\alpha(\delta, h)$ is the unique solution of (5.30), then we have

$$
\left\|\alpha^{2} R_{\alpha}^{2} A_{s}^{-s /(2 s+2 a)} L^{-s / 2} \tilde{y}\right\| \leq(b+\tilde{g}(s)) \delta+\left(d+\tilde{g}(s) c_{3}\|\hat{x}\|\right) h .
$$

Now the result follows from (5.33), (5.36), (5.37), and (5.38).

LEMMA 5.4. Suppose that $\hat{x}$ belongs to $H_{t}$ for some $t \leq s$, and $\alpha:=\alpha(\delta, h)>0$ is the unique solution of (5.30), where $b>\tilde{g}(s)$ and $d>c_{3}\|\hat{x}\| \tilde{g}(s)$ with $\tilde{g}(s):=g(-s /(2 s+2 a))$. Then

$$
\alpha \geq c_{0} \delta^{(s+a) /(t+a)}, \quad c_{0}=\frac{\min \left\{b-\tilde{g}(s), d-c_{3}\|\hat{x}\| \tilde{g}(s)\right\}}{g((s-2 t) /(2 s+2 a)) \rho}
$$


Proof. Note that by (5.23), Proposition 2.1, and (2.18), we have

$$
\begin{aligned}
F(\alpha, \tilde{y}) \leq & \alpha\left\|R_{\alpha} A_{s}^{-s /(2 s+2 a)} L^{-s / 2} P_{h} \tilde{y}\right\| \\
\leq & \alpha\left\|R_{\alpha} A_{s}^{-s /(2 s+2 a)} L^{-s / 2} P_{h}(\tilde{y}-y)\right\| \\
& +\alpha\left\|R_{\alpha} A_{s}^{-s /(2 s+2 a)} L^{-s / 2}\left(P_{h}-I\right) y\right\|+\alpha\left\|R_{\alpha} A_{s}^{-s /(2 s+2 a)} A_{s} L^{s / 2} \hat{x}\right\| \\
\leq & \alpha\left\|R_{\alpha} A_{s}^{-s /(2 s+2 a)} L^{-s / 2} P_{h}(\tilde{y}-y)\right\| \\
& +\alpha\left\|R_{\alpha} A_{s}^{-s /(2 s+2 a)} L^{-s / 2}\left(P_{h}-I\right) y\right\|+\alpha\left\|R_{\alpha} A_{s}^{(s+2 a) /(2 s+2 a)} L^{s / 2} \hat{x}\right\| \\
\leq & \alpha\left\|R_{\alpha} A_{s}^{-s /(2 s+2 a)} L^{-s / 2} P_{h}(\tilde{y}-y)\right\| \\
& +\alpha\left\|R_{\alpha} A_{s}^{-s /(2 s+2 a)} L^{-s / 2}\left(P_{h}-I\right) y\right\|+\alpha\left\|R_{\alpha} A_{s}^{(t+a) /(s+a)} A_{s}^{(s-2 t) /(2 s+2 a)} L^{s / 2} \hat{x}\right\| \\
\leq & \tilde{g}(s)\left[\delta+c_{3}\|\hat{x}\| h\right]+\left\|\alpha R_{\alpha} A_{s}^{(t+a) /(s+a)}\right\|\left\|A_{s}^{(s-2 t) /(2 s+2 a)} L^{s / 2} \hat{x}\right\| \\
\leq & \tilde{g}(s)\left[\delta+c_{3}\|\hat{x}\| h\right]+g\left(\frac{s-2 t}{2 s+2 a}\right) \rho \alpha^{(t+a) /(s+a)} .
\end{aligned}
$$

Thus

$$
\min \left\{b-\tilde{g}(s), d-c_{3}\|\hat{x}\| \tilde{g}(s)\right\}(\delta+h) \leq g\left(\frac{s-2 t}{2 s+2 a}\right) \rho \alpha^{(t+a) /(s+a)},
$$

which implies

$$
\alpha \geq c_{0}(\delta+h)^{(s+a) /(t+a)}, \quad c_{0}=\frac{\min \left\{b-\tilde{g}(s), d-c_{3}\|\hat{x}\| \tilde{g}(s)\right\}}{g((s-2 t) /(2 s+2 a)) \rho} .
$$

This completes the proof.

THEOREM 5.5. Under the assumptions in Lemma 5.4, for any fixed $\tau>0$,

$$
\left\|\tilde{x}_{\alpha}-\tilde{x}_{\alpha, h}\right\| \leq c_{5}(\delta+h)^{\zeta}
$$

where

$$
\zeta:=\frac{\tau}{\tau+1}+\frac{s}{2}-\frac{s+2 a}{2 t+2 a}+\gamma, \quad c_{5} \geq c c_{4} f\left(\frac{1}{2}\right)^{-s /(s+a)} \max \left\{g\left(\frac{1}{2}\right), 1\right\}
$$

with

$$
\gamma:= \begin{cases}0, & \text { if } t \geq 1-a, \\ \frac{s+a}{2}\left(1-\frac{1}{t+a}\right), & \text { if } t<1-a .\end{cases}
$$


Proof. Note that by (3.31) and Lemmas 5.3 and 5.4,

$$
\begin{aligned}
\| \tilde{x}_{\alpha}- & \tilde{x}_{\alpha, h} \| \\
\leq & f\left(\frac{1}{2}\right)^{-s /(s+a)} \Phi(s, h, \alpha) \alpha^{-a /(2 s+2 a)} h^{s}\left\|\tilde{x}_{\alpha}\right\|_{s} \\
\leq & c f\left(\frac{1}{2}\right)^{-s /(s+a)} \max \left\{g\left(\frac{1}{2}\right) h^{a / 2}, \alpha^{1 / 2} h^{-s / 2}\right\} c_{4} \alpha^{-a /(2 s+2 a)} h^{s} \alpha^{-1}(\delta+h)^{\tau /(\tau+1)} \\
\leq & c f\left(\frac{1}{2}\right)^{-s /(s+a)} \max \left\{g\left(\frac{1}{2}\right) h^{(s+a) / 2} \alpha^{-1 / 2}, 1\right\} c_{4} \alpha^{-a /(2 s+2 a)-1 / 2} h^{s / 2}(\delta+h)^{\tau /(\tau+1)} \\
\leq & c f\left(\frac{1}{2}\right)^{-s /(s+a)} \max \left\{g\left(\frac{1}{2}\right)(\delta+h)^{(s+a) / 2-(s+a) /(2 t+2 a)}, 1\right\} \\
& \times c_{4}(\delta+h)^{\tau /(\tau+1)+s / 2-a /(2 t+2 a)-(s+a) /(2 t+2 a)} \\
\leq & c f\left(\frac{1}{2}\right)^{-s /(s+a)} \max \left\{g\left(\frac{1}{2}\right), 1\right\} c_{4}(\delta+h)^{\tau /(\tau+1)+s / 2-a /(2 t+2 a)-(s+a) /(2 t+2 a)+\gamma .} .
\end{aligned}
$$

This completes the proof.

THEOREM 5.6. Under the assumptions in Lemma 5.4,

$$
\left\|\hat{x}-x_{\alpha}\right\|=O\left((\delta+h)^{t /(t+a)}\right)
$$

Proof. Since $x_{\alpha}$ is the solution of (2.22), we have

$$
\begin{aligned}
\hat{x}-x_{\alpha} & =\hat{x}-\left(A+\alpha L^{s}\right)^{-1} y \\
& =\alpha L^{-s / 2}\left(A_{s}+\alpha I\right)^{-1} L^{s / 2} \hat{x} \\
& =\alpha L^{-s / 2} R_{\alpha} L^{s / 2} \hat{x} .
\end{aligned}
$$

Therefore, by (5.7), we have

$$
f\left(\frac{s}{2 s+2 a}\right)\left\|\hat{x}-x_{\alpha}\right\| \leq\left\|\alpha A_{s}^{s /(2 s+2 a)} R_{\alpha} L^{s / 2} \hat{x}\right\|
$$

To obtain an estimate for $\left\|\alpha A_{s}^{s /(2 s+2 a)} R_{\alpha} L^{s / 2} \hat{x}\right\|$, first we will make use the moment inequality (2.17) with

$$
u=\frac{t}{a}, \quad v=1+\frac{t}{a}, \quad B=\alpha R_{\alpha} A_{s}^{a /(s+a)}, \quad x=\alpha^{1-t / a} R_{\alpha}^{1-t / a} A_{s}^{(s-2 t) /(2 s+2 a)} L^{s / 2} \hat{x}
$$


Then, since

$$
\|x\| \leq\left\|A_{s}^{(s-2 t) /(2 s+2 a)} L^{s / 2} \hat{x}\right\| \leq g\left(\frac{s-2 t}{2 s+2 a}\right)\left\|L^{s / 2} \hat{x}\right\|_{t-s / 2} \leq g\left(\frac{s-2 t}{2 s+2 a}\right) \rho,
$$

we have

$$
\begin{aligned}
& \left\|\alpha A_{s}^{s /(2 s+2 a)} R_{\alpha} L^{s / 2} \hat{x}\right\| \\
& \quad=\left\|B^{t / a} x\right\| \\
& \quad \leq\left\|B^{1+t / a} x\right\|^{t /(t+a)}\|x\|^{a /(t+a)} \\
& \quad \leq\left\|\alpha^{2} R_{\alpha}^{2} A_{s}^{(2 a+s) /(2 s+2 a)} L^{s / 2} \hat{x}\right\|^{t /(t+a)}\|x\|^{a /(t+a)} \\
& \quad \leq\left\|\alpha^{2} R_{\alpha}^{2} A_{s}^{-s /(2 s+2 a)} L^{-s / 2} y\right\|^{t /(t+a)}\|x\|^{a /(t+a)} \\
& \quad \leq g\left(\frac{s-2 t}{2 s+2 a}\right)^{a /(t+a)} \rho^{a /(t+a)}\left\|\alpha^{2} R_{\alpha}^{2} A_{s}^{-s /(2 s+2 a)} L^{-s / 2} y\right\|^{t /(t+a)} .
\end{aligned}
$$

Further, by (5.2), (5.7), and (5.21),

$$
\begin{aligned}
&\left\|\alpha^{2} R_{\alpha}^{2} A_{s}^{-s /(2 s+2 a)} L^{-s / 2} y\right\| \\
& \leq\left\|\alpha^{2} R_{\alpha}^{2} A_{s}^{-s /(2 s+2 a)} L^{-s / 2}(y-\tilde{y})\right\|+\left\|\alpha^{2} R_{\alpha}^{2} A_{s}^{-s /(2 s+2 a)} L^{-s / 2}\left(I-P_{h}\right) \tilde{y}\right\| \\
&+\left\|\alpha^{2} R_{\alpha}^{2} A_{s}^{-s /(2 s+2 a)} L^{-s / 2} P_{h} \tilde{y}\right\| \\
& \leq g\left(-\frac{s}{2 s+2 a}\right)\left(\delta+c_{3}\|\hat{x}\| h\right)+F(\alpha, \tilde{y}) .
\end{aligned}
$$

Therefore, if $\alpha:=\alpha(\delta, h)$ is the unique solution of (5.30), then we have

$$
\left\|\alpha^{2} R_{\alpha}^{2} A_{s}^{-s /(2 s+2 a)} L^{-s / 2} y\right\| \leq[\tilde{g}(s)+b] \delta+\left[\tilde{g}(s) c_{3}\|\hat{x}\|+d\right] h .
$$

Now the result follows from (5.49), (5.52), (5.53), and (5.54).

THEOREM 5.7. Under the assumptions in Lemma 5.4, for any fixed $\tau>0$,

$$
\left\|\hat{x}-\tilde{x}_{\alpha, h}\right\| \leq c_{6}(\delta+h)^{\mu}, \quad \mu:=\min \left\{\frac{t}{t+a}, \zeta\right\}
$$

for some $c_{6}>0$, and $\zeta$ as in Theorem 5.5. 
Proof. Let $x_{\alpha}$ and $\tilde{x}_{\alpha}$ be the solutions of (2.22) and (2.23), respectively. Then by triangle inequality, (5.4), and Proposition 2.1,

$$
\begin{aligned}
\| \hat{x} & -\tilde{x}_{\alpha, h} \| \\
& \leq\left\|\hat{x}-x_{\alpha}\right\|+\left\|x_{\alpha}-\tilde{x}_{\alpha}\right\|+\left\|\tilde{x}_{\alpha}-\tilde{x}_{\alpha, h}\right\| \\
& =\left\|\hat{x}-x_{\alpha}\right\|+\left\|L^{-s / 2} R_{\alpha} L^{-s / 2}(y-\tilde{y})\right\|+\left\|\tilde{x}_{\alpha}-\tilde{x}_{\alpha, h}\right\| \\
& \leq\left\|\hat{x}-x_{\alpha}\right\|+\frac{1}{f(s /(2 s+2 a))}\left\|A_{s}^{s /(2 s+2 a)} R_{\alpha} L^{-s / 2}(y-\tilde{y})\right\|+\left\|\tilde{x}_{\alpha}-\tilde{x}_{\alpha, h}\right\| \\
& \leq\left\|\hat{x}-x_{\alpha}\right\|+\frac{1}{f(s /(2 s+2 a))}\left\|A_{s}^{s /(s+a)} R_{\alpha} A_{s}^{-s /(2 s+2 a)} L^{-s / 2}(y-\tilde{y})\right\|+\left\|\tilde{x}_{\alpha}-\tilde{x}_{\alpha, h}\right\| \\
& \leq\left\|\hat{x}-x_{\alpha}\right\|+\frac{1}{f(s /(2 s+2 a))}\left\|A_{s}^{s /(s+a)} R_{\alpha}\right\|\left\|A_{s}^{-s /(2 s+2 a)} L^{-s / 2}(y-\tilde{y})\right\|+\left\|\tilde{x}_{\alpha}-\tilde{x}_{\alpha, h}\right\| \\
& \leq\left\|\hat{x}-x_{\alpha}\right\|+\frac{g(-s /(2 s+2 a))}{f(s /(2 s+2 a))} \delta \alpha^{-a /(s+a)}+\left\|\tilde{x}_{\alpha}-\tilde{x}_{\alpha, h}\right\| \\
& \leq\left\|\hat{x}-x_{\alpha}\right\|+\frac{g(-s /(2 s+2 a))}{f(s /(2 s+2 a))}(\delta+h) \alpha^{-a /(s+a)}+\left\|\tilde{x}_{\alpha}-\tilde{x}_{\alpha, h}\right\| .
\end{aligned}
$$

The proof now follows from Lemma 5.4 and Theorems 5.5 and 5.6.

COROLlary 5.8. If $t, s, a$ satisfy $\max \{0,1-a\}<t \leq s$ and $\tau$ is large enough such that

$$
\gamma+\frac{s}{2}\left[1-\frac{1}{t+a}\right] \geq \frac{1}{\tau+1}
$$

then

$$
\left\|\hat{x}-\tilde{x}_{\alpha, h}\right\| \leq c_{6}(\delta+h)^{t /(t+a)}
$$

with $c_{6}$ as in Theorem 5.7.

Proof. Let $\zeta, \mu$ be as in Theorems 5.5 and 5.7, respectively. Then we observe that

$$
\mu=\frac{t}{t+a} \quad \text { if } \gamma+\frac{s}{2}\left[1-\frac{1}{t+a}\right] \geq \frac{1}{\tau+1} .
$$

Hence the result follows from Theorem 5.7.

6. Order optimality of the error estimates. In order to measure the quality of an algorithm to solve an equation of the form (2.1), Micchelli and Rivlin [12] considered the quantity

$$
e(M, \delta):=\sup \{\|x\|: x \in M,\|A x\| \leq \delta\}
$$

and showed that

$$
e(M, \delta) \leq E(M, \delta) \leq 2 e(M, \delta)
$$


where

$$
E(M, \delta)=\inf _{R} \sup \{\|x-R v\|: x \in M, v \in H,\|A x-v\| \leq \delta\}
$$

is the best possible worst error. Here the stabilizing set $M$ is assumed to be convex such that $M=-M$ with $0 \in M$ (see also, Vaĭnikko and Veretennikov [22]), and infimum is taken over all algorithms $R: Y \rightarrow X$. Since $H$ is a Hilbert space and $A$ is assumed to be selfadjoint and positive, we, in fact, have (cf. Melkman and Micchelli [11])

$$
e(M, \delta)=E(M, \delta)
$$

Now using the assumption (2.18), and taking $r=-a, \lambda=0$ in the interpolation inequality (2.15), we obtain

$$
\|x\| \leq\|x\|_{-a}^{t /(t+a)}\|x\|_{t}^{a /(t+a)} \leq\left(\frac{\|A x\|}{c_{1}}\right)^{t /(t+a)}\|x\|_{t}^{a /(t+a)}, \quad x \in H_{t} .
$$

Therefore, for the set

$$
M_{t, \rho}=\left\{x:\|x\|_{t} \leq \rho\right\}
$$

with a fixed $t>0, \rho>0$,

$$
e\left(M_{t, \rho}, \delta\right) \leq\left(\frac{\delta}{c_{1}}\right)^{t /(t+a)} \rho^{a /(t+a)}
$$

It is known that the above estimate for $e\left(M_{t, \rho}, \delta\right)$ is sharp (cf. Vainikko [21]). In view of the above observations, an algorithm is called an optimal order yielding algorithm with respect to $M_{t, \rho}$ and the assumption (2.18), if it yields an approximation $\tilde{x}$ corresponding to the data $\tilde{y}$ with $\|y-\tilde{y}\| \leq \delta$ satisfying

$$
\|\hat{x}-\tilde{x}\|=O\left(\delta^{t /(t+a)}\right), \quad x \in H_{t} .
$$

Clearly, Corollary 5.8 shows that if $h=O(\delta)$ and if $\max \{0,1-a\}<t \leq s$ and $\tau$ is large enough such that

$$
\gamma+\frac{s}{2}\left[1-\frac{1}{t+a}\right] \geq \frac{1}{\tau+1},
$$

then we obtain the optimal order.

7. Applications. For $r \geq 2$, denote by $S_{h}$ the space of $r$ th-order splines on the uniform mesh of width $h=1 / n$, that is, $S_{h}$ consists of functions in $C^{r-1}[0,1]$ which are piecewise polynomials of degree $r-2$. For positive integers $s$, let $H^{s}$ denote the Sobolev space of functions $u \in C^{s-1}[0,1]$ with $u^{s-1}$ absolutely continuous and the norm $\|u\|_{H^{s}}$ defined by

$$
\|u\|_{H^{s}}=\left(\sum_{i=1}^{s}\left\|u^{(i)}\right\|\right)^{1 / 2}, \quad u \in H^{s}
$$


Then $S_{h}$ is a finite-dimensional subspace of $H^{r-1}$ which has the following well-known approximation property (cf. [1]): for $u \in H^{s}, s \in \mathbb{N}$, there is a constant $\kappa$ (independent of $h$ ) such that

$$
\inf _{\varphi \in S_{h}}\|u-\varphi\|_{H^{j}} \leq \kappa h^{\min \{s, r\}-j}\|u\|_{H^{s}}, \quad u \in H^{s}, j \in\{0,1\},
$$

so that assumption (2.32) is satisfied. We take $L$ as in (2.19), that is,

$$
L x:=\sum_{j=1}^{\infty} j^{2}\left\langle x, u_{j}\right\rangle u_{j}
$$

where $u_{j}(t):=\sqrt{2} \sin (j \pi t), j \in \mathbb{N}$, with domain of $L$ as

$$
D(L):=\left\{x \in L^{2}[0,1]: \sum_{j=1}^{\infty} j^{4}\left|\left\langle x, u_{j}\right\rangle\right|^{2}<\infty\right\} .
$$

In this case, $\left(H_{t}\right)_{t \in \mathbf{R}}$ is given as in (2.21). It can be seen that

$$
\begin{aligned}
H_{t} & =\left\{x \in L^{2}[0,1]: \sum_{j=1}^{\infty} j^{4 t}\left|\left\langle x, u_{j}\right\rangle\right|^{2}<\infty\right\} \\
& =\left\{x \in H^{2 t}(0,1): x^{(2 l)}(0)=x^{(2 l)}(1)=0, l=0,1, \ldots,\left[t-\frac{1}{4}\right\rceil\right\},
\end{aligned}
$$

where $\lceil p\rceil$ denotes the greatest integer less than or equal to $p$. We observe that $H^{0}=$ $L^{2}[0,1]$, and for $s \in \mathbb{N}, H_{s} \subset H^{s}$.

Now, let $A: L^{2}[0,1] \rightarrow L^{2}[0,1]$ be a positive selfadjoint operator. Then we have

$$
\left\|A\left(I-P_{h}\right)\right\|=\|(I-P) A\|=\sup _{\|u\| \leq 1} \inf _{\varphi \in S_{h}}\|A u-\varphi\| .
$$

Hence, by (7.2),

$$
\left\|A\left(I-P_{h}\right)\right\| \leq \kappa h^{\min (s, r)} \sup _{\|u\| \leq 1}\|A u\|_{H^{s}}
$$

From the above inequality it is clear that if $A u \in H_{s}$ for every $u \in L^{2}[0,1]$, and if $A: L^{2}[0,1] \rightarrow H_{s}$ is a bounded operator, then there exists a constant $\hat{c}$ such that

$$
\left\|A\left(I-P_{h}\right)\right\| \leq \kappa \hat{c} h^{\min (s, r)}
$$

so that (5.2) is satisfied.

Now, we consider the case of an integral operator, namely (2.4), having all its eigenvalues nonnegative, and $k(\xi, t)=k(t, \xi)$ for all $(\xi, t) \in[0,1] \times[0,1]$ is such that it is differentiable $s$ times with respect to the variable $\xi$ with its $s$ th derivative lying in $L^{2}[0,1]$. For example, the integral operator may be the one associated with the backward heat equation problem considered in Section 2.

Now,

$$
\frac{d^{i}}{d \xi^{i}}(A u)(\xi)=\int_{0}^{1} \frac{\partial^{i} k(\xi, t)}{\partial \xi^{i}} u(t) d t
$$


so that

$$
\|A u\|_{H^{s}} \leq\|k\|_{0, s}\|u\| \quad \text { with }\|k\|_{0, s}=\sum_{i=0}^{s} \int_{0}^{1} \int_{0}^{1}\left|\frac{\partial^{i} k(t, \xi)}{\partial \xi^{i}}\right|^{2} d t d \xi .
$$

Thus we get (7.8) with $\hat{c}=\|k\|_{0, s}$.

\section{REFERENCES}

[1] I. Babuška and A. K. Aziz, Survey lectures on the mathematical foundations of the finite element method, The Mathematical Foundations of the Finite Element Method with Applications to Partial Differential Equations (Proc. Sympos., Univ. Maryland, Baltimore, Md, 1972) (A. K. Aziz, ed.), Academic Press, New York, 1972, pp. 1-359.

[2] H. W. Engl, M. Hanke, and A. Neubauer, Regularization of Inverse Problems, Mathematics and Its Applications, vol. 375, Kluwer Academic Publishers, Dordrecht, 1996.

[3] H. W. Engl and A. Neubauer, Convergence rates for Tikhonov regularization in finitedimensional subspaces of Hilbert scales, Proc. Amer. Math. Soc. 102 (1988), no. 3, 587-592.

[4] S. George and M. T. Nair, Error bounds and parameter choice strategies for simplified regularization in Hilbert scales, Integral Equations Operator Theory 29 (1997), no. 2, 231-242.

[5] _ An optimal order yielding discrepancy principle for simplified regularization of illposed problems in Hilbert scales, Int. J. Math. Math. Sci. (2003), no. 39, 2487-2499.

[6] C. W. Groetsch, The Theory of Tikhonov Regularization for Fredholm Equations of the First Kind, Research Notes in Mathematics, vol. 105, Pitman, Massachusetts, 1984.

[7] M. Hegland, An optimal order regularization method which does not use additional smoothness assumptions, SIAM J. Numer. Anal. 29 (1992), no. 5, 1446-1461.

[8] A. Kirsch, An Introduction to the Mathematical Theory of Inverse Problems, Applied Mathematical Sciences, vol. 120, Springer-Verlag, New York, 1996.

[9] S. G. Krein and J. I. Petunin, Scales of Banach spaces, Russian Math. Surveys 21 (1966), 85-160.

[10] B. A. Mair, Tikhonov regularization for finitely and infinitely smoothing operators, SIAM J. Math. Anal. 25 (1994), no. 1, 135-147.

[11] A. A. Melkman and C. A. Micchelli, Optimal estimation of linear operators in Hilbert spaces from inaccurate data, SIAM J. Numer. Anal. 16 (1979), no. 1, 87-105.

[12] C. A. Micchelli and T. J. Rivlin, A survey of optimal recovery, Optimal Estimation in Approximation Theory (C. A. Micchelli and T. J. Rivlin, eds.), Plenum Press, New York, 1977, pp. 1-54.

[13] M. T. Nair, On Morozov's method for Tikhonov regularization as an optimal order yielding algorithm, Z. Anal. Anwendungen 18 (1999), no. 1, 37-46.

[14] _ Functional Analysis: A First Course, Prentice Hall of India Private Limited, New Delhi, 2002.

[15] _ Optimal order results for a class of regularization methods using unbounded operators, Integral Equations Operator Theory 44 (2002), no. 1, 79-92.

[16] M. T. Nair, M. Hegland, and R. S. Anderssen, The trade-off between regularity and stability in Tikhonov regularization, Math. Comp. 66 (1997), no. 217, 193-206.

[17] F. Natterer, Error bounds for Tikhonov regularization in Hilbert scales, Applicable Anal. 18 (1984), no. 1-2, 29-37.

[18] A. Neubauer, An a posteriori parameter choice for Tikhonov regularization in Hilbert scales leading to optimal convergence rates, SIAM J. Numer. Anal. 25 (1988), no. 6, 13131326.

[19] E. Schock, Ritz-regularization versus least-square-regularization. Solution methods for integral equations of the first kind, Z. Anal. Anwendungen 4 (1985), no. 3, 277-284. 
[20] T. Schröter and U. Tautenhahn, Error estimates for Tikhonov regularization in Hilbert scales, Numer. Funct. Anal. Optim. 15 (1994), no. 1-2, 155-168.

[21] G. Vainikko, On the optimality of methods for ill-posed problems, Z. Anal. Anwendungen 6 (1987), no. 4, 351-362.

[22] G. M. Vaŭnikko and A. Y. Veretennikov, Iteration Procedures in Ill-Posed Problems, 1st ed., Nauka, Moscow, 1986.

[23] H. F. Weinberger, A First Course in Partial Differential Equations with Complex Variables and Transform Methods, Blaisdell Publishing, New York, 1965.

Santhosh George: Department of Mathematics, Government College of Arts, Science and Commerce, Sanquelim, Goa 403505, India

E-mail address: santhoshsq1729@yahoo.co.in

M. Thamban Nair: Department of Mathematics, Indian Institute of Technology Madras, Chennai 600036, India

E-mail address: mtnair@i itm.ac.in 


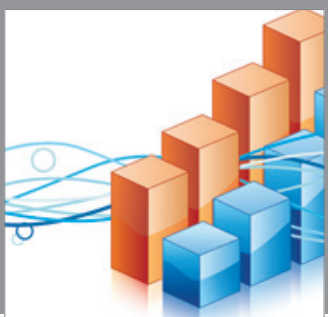

Advances in

Operations Research

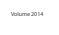

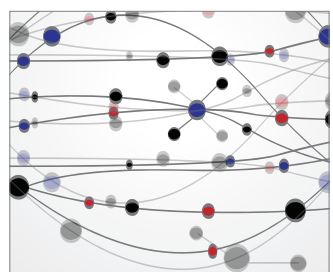

\section{The Scientific} World Journal
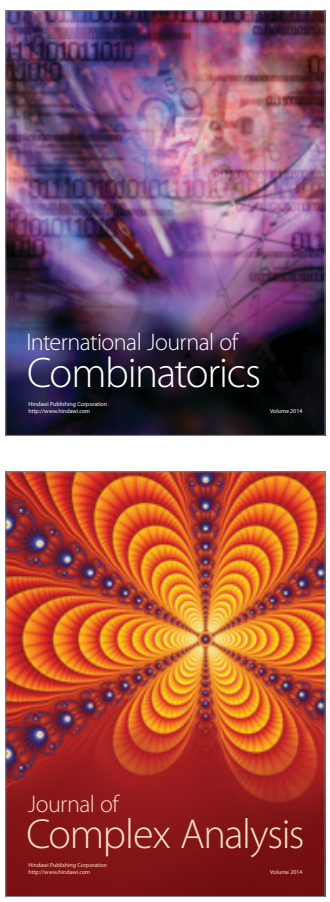

International Journal of

Mathematics and

Mathematical

Sciences
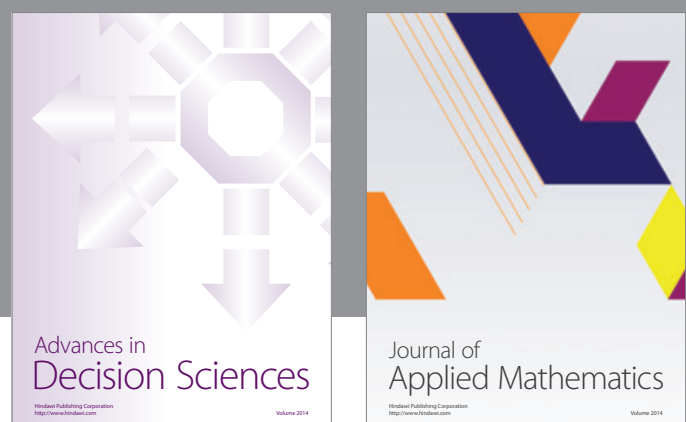

Journal of

Applied Mathematics
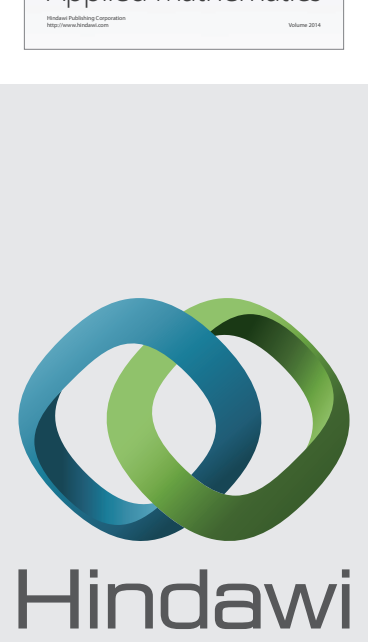

Submit your manuscripts at http://www.hindawi.com
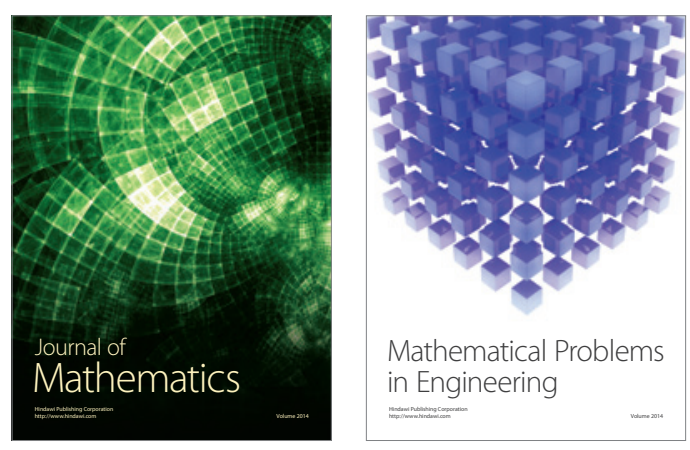

Mathematical Problems in Engineering
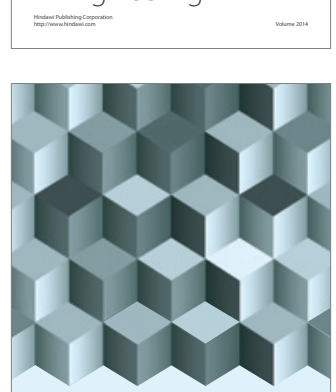

Journal of

Function Spaces
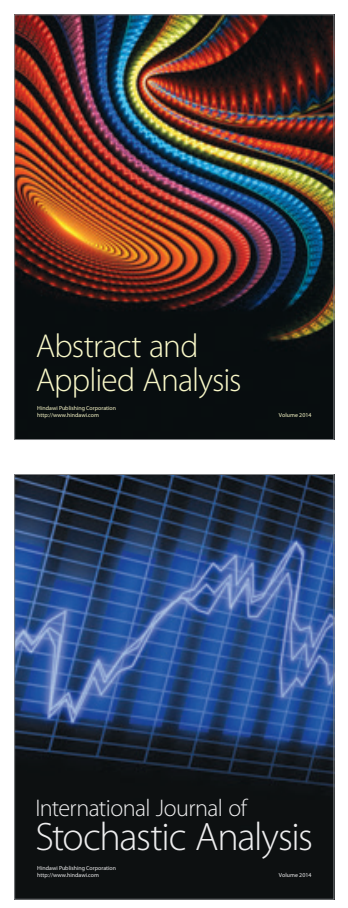

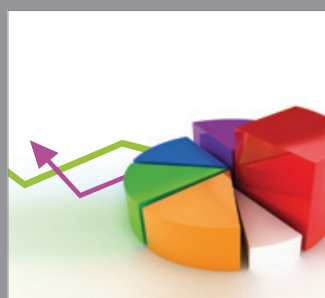

ournal of

Probability and Statistics

Promensencen
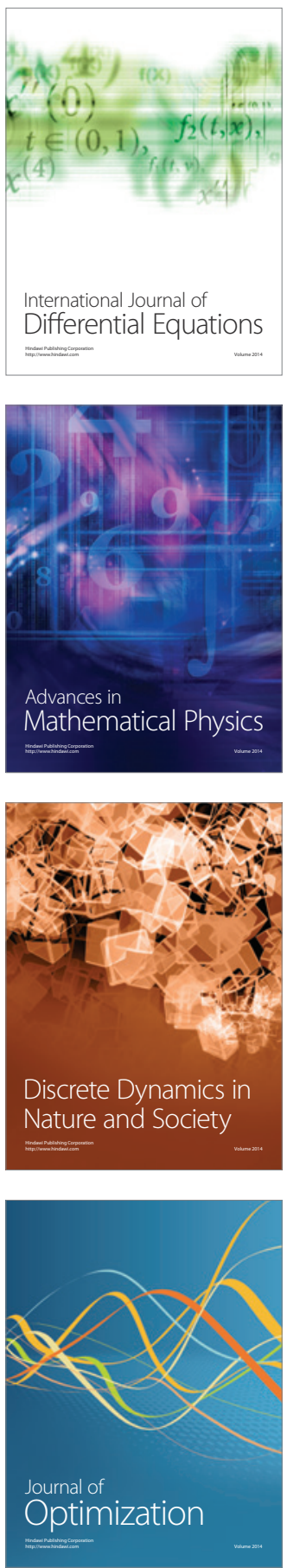